\title{
A case of chest pain and heart failure
}

\author{
Emanuela Scannella $\cdot$ Laura Angaroni · \\ Anna Coerezza - Monica Solbiati · Fabrizio Foieni • \\ Nicola Montano
}

Received: 28 March 2011 / Accepted: 12 April 2011/Published online: 6 May 2011

(c) SIMI 2011

\section{Case presentation}

Dr Montano: A 60-year-old-man was admitted to our Emergency Department (ED) for worsening dyspnea, and recurrent episodes of oppressive chest pain that resolved within a few minutes with rest. He had a history of benign prostatic hypertrophy (in therapy with Finasteride $5 \mathrm{mg} /$ day), mild chronic renal failure, a prior episode of right lower limb thrombophlebitis (1992), pulmonary sarcoidosis diagnosed in 1991 and initially treated with 6 months of steroid therapy without further follow-up in the past few years. His history was negative for smoking or other cardiovascular risk factors (Fig. 1).

In ED, he appeared diaphoretic and dyspnoeic, was tachycardic and his blood pressure was 190/120 mmHg. The physical examination was otherwise substantially normal. An ECG showed sinus tachycardia (125 bpm), and isolated premature ventricular beats. The arterial blood gas showed respiratory failure with a normal $\mathrm{pCO}_{2}$ level. The blood tests evidenced a slight increase in T-troponin (22 ng/L, $52 \mathrm{ng} / \mathrm{l} 6 \mathrm{~h}$ later) and D-dimer (841 ng/dL), and confirmed the known mild renal failure (creatinine $1.38 \mathrm{mg} / \mathrm{dL}$ ). A chest X-ray study was comparable to previous monitoring showing the known pattern of hilaradenopathy and reticular opacities.

The patient was then treated with captopril, nitroglycerine and furosemide iv. A second ECG performed $3 \mathrm{~h}$ later showed a sinus tachycardia (145 bpm), and a left

E. Scannella $(\varangle) \cdot$ L. Angaroni · A. Coerezza - M. Solbiati ·

F. Foieni $\cdot$ N. Montano

Dipartimento di Scienze Cliniche L. Sacco, Università degli

Studi di Milano, Ospedale Luigi Sacco, Via G.B. Grassi 74,

20157 Milan, Italy

e-mail: emanuelascannella@gmail.com bundle branch block of new onset. At the end of the infusional therapy, reduction in arterial blood pressure $(140 / 80 \mathrm{mmHg})$ and in heart rate $(110 / \mathrm{min})$ were obtained, and the patient reported subjective improvement in symptoms. He was admitted to our department with a diagnosis of heart failure and hypertensive crisis.

At the time of hospital admission, arterial blood pressure and heart rate were still high (BP 170/105 $\mathrm{mmHg}, \mathrm{HR}$ 126 beats/min), the patient was dyspnoeic at rest without chest pain.

\section{Differential diagnosis}

Dr Angaroni, Dr Coerezza, Dr Scannella: The presence of worsening dyspnoea, regressed with reduction in blood pressure and heart rate, and associated with a radiological picture compatible with overloading of the pulmonary circulation, is suggestive for acute congestive heart failure rather than for a pulmonary cause of dyspnoea.

Although this clinical picture might have been triggered by an hypertensive crisis or a run of tachycardia, in the presence of a new left bundle branch block, the hypothesis of an acute ischemic origin of the heart failure should be considered [1]. A coronary angiogram should be performed as soon as possible; however, since at the moment of the first evaluation in our department, the left bundle branch block had appeared more than $24 \mathrm{~h}$ prior, we could delay the coronary study, and start a medical treatment.

In consideration of the rapid onset of the dyspnoea, associated with the positivity of $\mathrm{D}$-dimer and the respiratory failure with a normal $\mathrm{pCO}_{2}$ level on the arterial blood gas, the hypothesis of pulmonary embolism should also be considered. The pre-test clinical probability in this patient was intermediate according to the Geneva score [2] 


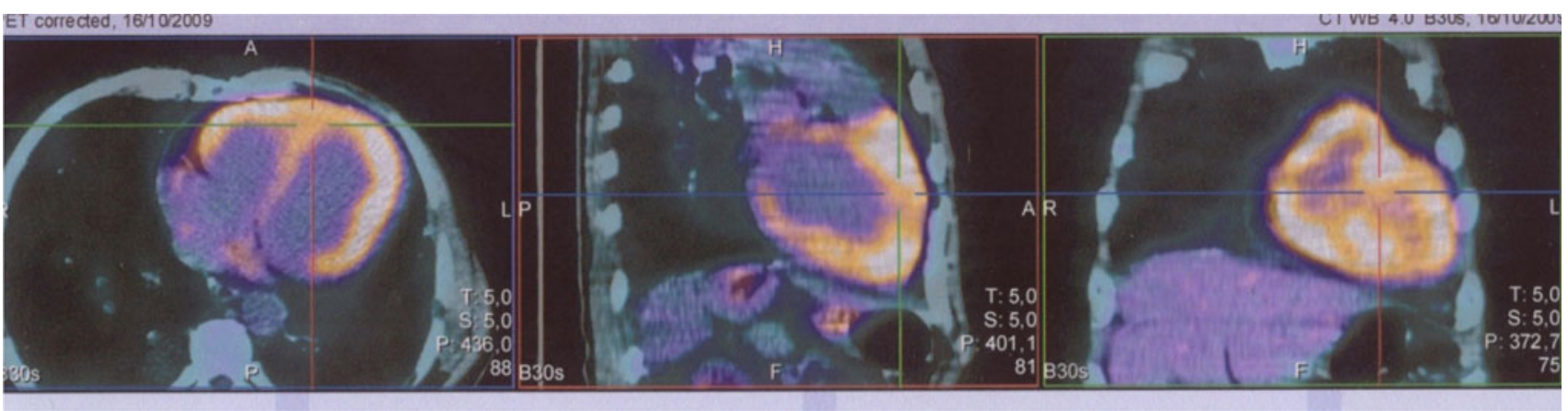

Fig. 1 18-FDG PET images showing widespread uptake of the tracer in the myocardium

(HR $>95 \mathrm{bpm})$ and low according to the Wells' score [3] $(\mathrm{HR}>100 \mathrm{bpm})$. Thus, in agreement with the European guidelines, an angiographic-TC scan should be performed [4]. The ischemic hypothesis was however much stronger, and in the presence of a new onset left bundle branch block it must be considered as the most likely one. In addition, there was no urgency to perform an angiographic-TC scan after anticoagulant therapy had been started.

\section{Preliminary diagnosis}

Dr Solbiati, Dr Scannella: The treatment with lowmolecular weight heparin, aspirin, clopidogrel, intravenous nitrates, carvedilol and ACE inhibitors was immediately started, achieving a reduction in blood pressure and heart rate, and a subjective improvement of symptoms.

A Doppler-echocardiography was performed, showing widespread and severe hypokinesia, severe reduction in pump function (ejection fraction $27 \%$ ), impaired left ventricular diastolic function and pulmonary hypertension.

To evaluate a possible underlying coronary artery disease, a coronary angiogram was performed without evidence of significant stenosis. Furthermore, the left bundle branch block disappeared with the reduction in heart rate.

\section{Further investigations}

Dr Montano, Dr Foieni: Because the acute heart failure and the echocardiographic finding of hypokinetic cardiomyopathy could not be attributed to an acute ischemic event, we decided to stop the anti-ischemic therapy based on the nitrates, anticoagulant and antiplatelet drugs to evaluate other hypotheses.

Considering the patient's history, we then hypothesized an etiological correlation between the previous diagnosis of sarcoidosis and the present cardiac clinical picture.

In fact, a myocardial involvement should be considered in the evaluation of a patient with known sarcoidosis who develops heart failure, dysrhythmias or conduction disease [5].

The hypothesis of an association between acute heart failure and sarcoidosis was therefore advanced.

We decided to perform a PET scan, which has a high sensitivity in the detection of the earlier stages of myocardial sarcoidosis [5], which showed an intense and widespread uptake of the tracer in the heart, as well as a bilateral hilar hyperconcentration.

\section{Diagnosis and therapy}

Dr Solbiati, Dr Montano: This pattern of widespread hyperconcentration both in heart and lungs, associated with a history of sarcoidosis, was strongly suggestive for cardiac sarcoidosis. Further examinations excluded the involvement of other organs.

We continued the therapy with beta-blockers and ACE inhibitors, and started high dosage glucocorticoids $(1 \mathrm{mg} / \mathrm{kg}$ ) administration, to attenuate the inflammatory response.

Considering the highly depressed systolic function and the risk of malignant cardiac dysrhythmias, we proposed to the patient the insertion of an implantable cardioverterdefibrillator (ICD), which however he refused.

The patient was then discharged in good hemodynamic condition.

At a 3-month follow-up visit, an echocardiogram showed an improvement in systolic function with a significant increase in eject fraction to $38 \%$.

\section{Discussion}

Dr Angaroni, Dr Coerezza, Dr Scannella: Sarcoidosis is a chronic, multisystem disease characterized histologically by the formation of granulomas in many tissues. Clinical evidence of myocardial involvement is present in approximately $5 \%$ of patients affected by sarcoidosis, and may 
precede, follow, or occur concurrently with involvement of the lungs or other organs [6].

The clinical presentation of cardiac sarcoidosis depends on the presence and extent of granulomas and fibrous tissue within the myocardium, in particular in the conduction system, resulting in alterations in conduction, contractility and relaxation. It may range from a benign, incidentally discovered condition to a life-threatening disorder, such as sudden death due to ventricular tachydysrhythmias or conduction block. The presence of cardiac sarcoidosis should be raised in any patient with known sarcoidosis who develops dysrhythmias, conduction disturbances or cardiovascular failure.

No single diagnostic test has emerged that combines a high degree of sensitivity and specificity in the diagnosis of cardiac sarcoidosis. Traditionally, an endomyocardial biopsy is considered the gold standard; however, due to its invasive nature and its low sensitivity, imaging methods are currently preferred both for the diagnosis and follow-up of a patient with suspected cardiac sarcoidosis [7]. These include Thallium-201 or Gallium-67 radionuclide testing, positron emission tomography (PET) with fluoro-2-deoxyD-glucose (18-FDG), and gadolinium-enhanced magnetic resonance imaging (MRI). Although there are limited data available to define their sensitivity, specificity, or predictive value, 18-FDG PET seems to combine a higher sensitivity (ranging from 82 to $99 \%$ ) with the possibility of performing a total body study, despite a relatively low specificity [8].

Multiple studies support the efficacy of treatment with corticosteroids, even if their effects on the clinical course of cardiac sarcoidosis have not been studied in large, randomized trials. Definitive data on efficacy, optimal dose and duration are lacking. Some authors suggest an initial high dose (e.g. prednisone $60 \mathrm{mg} /$ day) and a subsequent reduction with regular clinical monitoring [7-9].
Acknowledgments This case record was selected for the "CasiClinici Gymnasium" session at the 2010 SIMI Annual Congress in Rome, and was reviewed by a Committee composed of Maria Domenica Cappellini and Luigi Pagliaro.

Conflict of interest None.

\section{References}

1. Artman EM, Hand M, Armstrong PW et al (2008) 2007 Focused update of the ACC/AHA 2004 guidelines for the management of patients with ST-elevation myocardial infarction: a report of the American College of Cardiology/American Heart Association Task Force on Practice Guidelines: developed in collaboration with the Canadian Cardiovascular Society endorsed by the American Academy of Family Physicians: 2007 Writing Group to Review New Evidence and Update the ACC/AHA 2004 Guidelines for the Management of Patients with ST-Elevation Myocardial Infarction, Writing on Behalf of the 2004 Writing Committee

2. Le Gal G, Righini M, Roy PM et al (2006) Prediction of pulmonary embolism in the emergency department: the revised Geneva score. Ann Inter Med 144:165-171

3. Wells PS, Anderson DR, Rodger M et al (2000) Derivation of a simple clinical model to categorize patients probability of pulmonary embolism: increasing the models utility with the SimpliRED D-dimer. Thromb Haemost 83:416-420

4. Torbicki A, Chairperson PerrierA et al (2008) Guidelines on the diagnosis and management of acute pulmonary embolism. Eur Heart J 29:2276-2315

5. Sharma S (2009) Cardiac imaging in myocardial sarcoidosis and other cardiomyopathies. Curr Opin Pulm Med 15:507-512

6. Chapelon-Abric C, De Zuttere D, Duhaut P et al (2004) Cardiac sarcoidosis: a retrospective study of 41 cases. Medicine 83:315-319

7. Habersberger J, Manins V, Taylor AJ (2008) Cardiac sarcoidosis. Intern Med J 38:270-277

8. Kim JS, Judson MA, Donnino R et al (2009) Cardiac sarcoidosis. Am Heart J 157:9-21

9. Simon W, Dubrey RodneyH et al (2010) Diagnosis and management of cardiac sarcoidosis. Prog Cardiovasc Dis 52:336-346 